\title{
Effect of The "Normalized Epidemic Prevention And Control Requirements" On Hospital-Acquired And Community-Acquired Infections In China
}

\section{Caiyun Chen}

The First Affiliated Hospital of Nanjing Medical University

\section{Ping Zhu}

The First Affiliated Hospital of Nanjing Medical University

\section{Yongxiang Zhang}

The First Affiliated Hospital of Nanjing Medical University

\section{Bo Liu ( $\nabla$ liusanbo@163.com )}

The First Affiliated Hospital of Nanjing Medical University

\section{Research Article}

Keywords: "Normalized Epidemic Prevention and Control Requirements", epidemic prevention and control; Hospital-acquired infection; Infectious sites; Multi-drug resistant organisms; Usage of antibiotics

Posted Date: September 27th, 2021

DOI: https://doi.org/10.21203/rs.3.rs-916149/v1

License: (c) (i) This work is licensed under a Creative Commons Attribution 4.0 International License. Read Full License

Version of Record: A version of this preprint was published at BMC Infectious Diseases on November 23rd, 2021. See the published version at https://doi.org/10.1186/s12879-021-06886-y. 


\section{Abstract}

Background: No studies have yet reported the effect of prevention and control measures, which were implemented to combat COVID-19, on the prevention and control of common HAls. We aimed to examine the effect of the "Normalized Epidemic Prevention and Control Requirements" (implemented in May 2020) by comparison of hospital-acquired infections (HAls) and community-acquired infections (CAls) in China during 2018, 2019, and 2020.

Methods: Data of inpatients before and after implementation of new requirements were retrospectively analyzed, including infection rate, use of alcohol-based hand cleaner, anatomical sites of infections, pathogen species, infection by multi-drug-resistant species, use of different antibiotics, and antibiotic use density.

Results: The HAl rate was significantly higher in 2020 than in 2018 and $2019(P<0.05)$, and the CAl rate was significantly higher in 2019 and 2020 than in $2018(P<0.001)$. Lower respiratory tract infections were the most common HAI during all years, with no significant changes over time. Lower respiratory tract infections were also the most common CAl, but were significantly more common in 2018 and 2019 than $2020(P<0.001)$. There were no changes in upper respiratory tract infections among HAls or CAls. Most HAls and CAls were from Gram-negative bacteria, and the percentages of fungal infections were greater in 2019 and 2020 than 2018. MRSA infections were more common in 2020 than in 2018 and 2019 $(P<0.05)$. The utilization rate and usage days of antibiotics decreased over time $(P<0.001)$, the culture rate of microbial specimens before antibiotics usage increased over time $(P<0.001)$, but antibiotic use density remained steady over time.

Conclusions: The new prevention and control requirements provided important benefits during the COVID19 pandemic. However, their effects on HAls were not obvious.

\section{Background}

During December 2019, many cases of pneumonia of unknown cause appeared in Wuhan (Hubei Province, China). This disease soon spread to other regions of China and then around the world, becoming a pandemic [1]. The World Health Organization (WHO) declared COVID-19 was a Public Health Emergency of International Concern on 1 February 2020 [2]. Researchers identified the causative pathogen was a species in the Betacoronavirus genus that was distinct from other well-known species in this genus, SARS-CoV and MERS-CoV [3-4]. The World Committee on the Classification of Viruses named this novel virus as severe acute respiratory syndrome coronavirus 2 (SARS-CoV-2) and the WHO named the disease as coronavirus disease 2019 (COVID-19) [5].

It is now established that the virus has a high rate of transmission, and this motivated China to implement stringent prevention and control measures. As of 27 August 2021, there have been more than 2.14-billion confirmed cases of COVID-19 and more than 4.47-million deaths worldwide [6]. Although many countries continue to suffer from this serious pandemic, China has been successful in controlling 
COVID-19, although it had about 100 thousand cases and more than 4000 deaths in total. With the cessation of the blockade in Wuhan, there was increased focus on prevention and control to prevent imported cases and a rebound of indigenous cases in China. Based on risk assessments, targeted methods of prevention and control were implemented that promoted the resumption of work and production [7].

Hospitals are densely populated places and many patients are immunocompromised, increasing the risk of cross-infection. Hospitals are also necessary for the care of people with severe infections and have a key function in prevention and control during pandemics. Therefore, health administrators in China required hospitals to implement "Normalized Epidemic Prevention and Control Requirements" during May 2020 to improve prevention and control measures [8], such as management of hospitalization, management of the family members of patients, management of visitors, and other policies. These requirements have been in place for more than 1 year. In addition to prevention and control of the COVID19 pandemic, prevention and control of common hospital-acquired infections (HAls) was also a focus of these prevention efforts. However, no studies have yet reported the effect of these requirements, which were implemented to combat COVID-19, on the prevention and control of common HAls. In this study, we examined the relationship of the implementation of the "Normalized Epidemic Prevention and Control Requirements" on HAls and community-acquired infections (CAls).

\section{Materials And Methods}

The records of hospitalized patients from 1 May to 31 December 312020 (study group) and from the same periods of 2018 and 2019 (control group) were retrospectively analyzed. All study subjects were patients at the First Affiliated Hospital with Nanjing Medical University, Nanjing. Data were extracted from the nosocomial infection real time surveillance system. Diagnosis of standard of HAls was according to the definition of the U.S. Centers for Disease Control and Prevention (CDC) [9]. Ethical approval for this retrospective study was obtained from the local ethics committee of the First Affiliated Hospital with Nanjing Medical University (2021-SR-152).

Normalized Epidemic Prevention and Control Requirements

Numerous requirements for prevention and control of the COVID-19 pandemic were implemented in 1 May 2020. In particular, body temperature and travel records were required for all individuals before entering a hospital, and all individuals were also required to wear masks when entering a hospital. Clinics were required to comply with the principle of one patient and one healthcare worker per room. Each hospitalized patient was accompanied by one dedicated person. Hospitalized patients were educated by healthcare workers regarding methods to be used for disease prevention and control. Hospitalized patients with accompanying persons were generally required to wear masks in the ward, and visitors were not allowed to enter. All healthcare workers were required to wear surgical masks during routine medical procedure. In addition, an expert preventionist trained healthcare workers regarding methods to be used to prevent and control infections two times per month. 


\section{Identification of microorganism and standards of multi-drug resistant organism}

Bacterial culture and species identification were performed according to the National Clinical Laboratory Operating Procedures (Fourth Edition) [10]. Pure cultured strains were analyzed using an automated bacterial identification instrument (Vitek COMPACT 2, France, bioMérieuxe), identified using supporting identification cards, and the results were reported according to the requirements of the Clinical Laboratory Standards Institute (CLSI) [11]. Multi-drug resistant organisms (MDROs) were defined according to the 2015 consensus of Chinese experts on the prevention and control of HAls by multi-drug resistant organisms [12]. These MDROs mainly consisted of methicillin-resistant Staphylococcus aureus (MRSA), vancomycin-resistant Enterococcus (VRE), carbapenem-resistant Enterobacteriaceae (CRE), carbapenemresistant Acinetobacter baumannii (CR-AB), and carbapenem-resistant Pseudomonas aeruginosa (CRPA). The control strains used for quality assurance (Escherichia coli ATCC 25922, P. aeruginosa ATCC 27853 , and S. aureus ATCC 25923) were provided by the clinical testing center of the National Health Commission. Colonization and contamination by pathogens were actively prevented, and duplicate strains from the same site of one patient were removed from analysis.

\section{Antibiotic use density}

The antibiotic use density (AUD) was calculated from the sum of defined daily doses (DDDs) of common antibiotics issued by the WHO [13] and the number of inpatient days: AUD $=($ DDDs $\times 100) /$ inpatient days.

\section{Research contents}

The infection rate, use of quick-drying hand disinfectant, anatomical sites of infection, species of infectious pathogens, HAI rate by MDROs, use of therapeutic antibiotics, and changes in AUD were compared for the study and control groups.

\section{Statistical analysis}

A database was established using Excel 2010, and the data were then analyzed using SPSS version 23.0 (SPSS Inc., Chicago, IL). Enumeration data were presented as rate and ratio and analyzed using the $\chi^{2}$ test or the exact probability method. Measurement data were presented as means \pm SDs if it had a normal distribution and as median with interquartile range (IQR) if it had a non-normal distribution. The two groups were compared using the nonparametric Mann-Whitney $U$ test, and comparisons of distributions were performed using the nonparametric Kruskal-Wallis $\mathrm{H}$ test. A $P$-value below 0.05 was considered significant.

\section{Results}

\section{Infection rates before and after the intervention}


There were 62,625 inpatients (48.25\% males, $51.75 \%$ females) in $2018,70,091$ inpatients ( $48.50 \%$ males, $51.50 \%$ females) in 2019 , and 59,167 inpatients ( $48.52 \%$ males, $51.48 \%$ females) in 2020 , and their average ages were $52 \pm 19$ years (2018), $53 \pm 18$ years (2019), and $53 \pm 19$ years (2020). There were no significant differences in these gender ratios or ages. However, the numbers of HAls during 2020 was greater than during 2019 or 2018 (both $P<0.05$ ) and the numbers of CAls during 2020 was greater than during 2019 or 2018 (both $P<0.001$ ) (Table 1).

Table 1

Percentages of inpatients with hospital-acquired infections and communityacquired infections among all inpatients from 2018 to $2020^{*}$

\begin{tabular}{|lll|}
\hline Year & Hospital-acquired infections (\%) & Community-acquired infections (\%) \\
\hline 2018 & $1.64 \%(1024 / 62,625)$ & $2.66 \%(1664 / 62,625)$ \\
\hline 2019 & $1.56 \%(1112 / 71,190)$ & $3.18 \%(2266 / 71,190)$ \\
2020 & $1.82 \%(1077 / 59,167)$ & $3.33 \%(1972 / 59,167)$ \\
\hline$\chi^{2}$ & 13.666 & 52.791 \\
$P$ & 0.001 & $<0.001$ \\
\hline$*$ Numbers in parentheses indicate infected inpatients/total inpatients. \\
\hline
\end{tabular}
ges in Open beds and USe of alcohol-based hand
P

The total number of open bed days was 3757 in 2018, 3988 in 2019, and 3820 in 2020, and this corresponded to 8.13 open beds per day in 2018, 11.69 open beds per day in 2019, and 13.75 open beds per day in 2020 (Fig. 1). The total consumption of alcohol-based hand cleaner increased during this time from $3575 \mathrm{~mL}$ in 2018, to $5733 \mathrm{~mL}$ in 2019, and to $6461 \mathrm{~mL}$ in 2020 .

\section{Changes in infection sites}

Analysis of the anatomical sites of HAls indicated the lower respiratory tract was the most common site during all 3 years, with no significant difference among the years (Table 2). However, the percentage of urinary system infections was significantly lower during 2020 than $2018(P=0.002)$ and $2019(P=$ 0.001). The percentage of eye/ear/nose/throat/mouth infections was greater during 2020 than 2018 ( $P=$ 0.004), but the percentages were similar for 2019 and 2020. Except for infections at "other site" (which were rare during all 3 years), there were no other significant differences. 
Table 2

Anatomical sites of hospital-acquired infections from 2018 to $2020^{*}$

\begin{tabular}{|llllll|}
\hline Infection site & $\mathbf{2 0 1 8}$ & $\mathbf{2 0 1 9}$ & $\mathbf{2 0 2 0}$ & $\mathbf{\chi}^{\mathbf{2}}$ & $\boldsymbol{P}$ \\
\hline Lower respiratory tract & $39.22 \%$ & $39.51 \%$ & $39.70 \%$ & 0.059 & 0.971 \\
\hline Urinary system & $14.58 \%$ & $14.83 \%$ & $10.51 \%$ & 12.681 & $\mathbf{0 . 0 0 2}$ \\
\hline Blood system & $14.32 \%$ & $11.28 \%$ & $12.91 \%$ & 5.083 & 0.079 \\
\hline Surgical site & $13.13 \%$ & $11.83 \%$ & $14.84 \%$ & 4.965 & 0.084 \\
\hline Abdomen and digestive system & $7.33 \%$ & $7.49 \%$ & $9.62 \%$ & 5.390 & 0.068 \\
\hline Upper respiratory tract & $5.46 \%$ & $4.89 \%$ & $3.85 \%$ & 3.698 & 0.165 \\
\hline Skin and soft tissue & $3.58 \%$ & $4.26 \%$ & $2.81 \%$ & 3.873 & 0.144 \\
\hline Eye/ear/nose/throat/mouth & $0.94 \%$ & $1.66 \%$ & $2.49 \%$ & 8.660 & $\mathbf{0 . 0 1 3}$ \\
\hline Genital tract & $0.43 \%$ & $0.08 \%$ & $0.24 \%$ & 0.807 & 0.369 \\
\hline Central nervous system & 0 & $0.79 \%$ & 0 & - & - \\
\hline Bone and joint & 0 & $0.16 \%$ & $0.16 \%$ & - & - \\
\hline Cardiovascular system & 0 & $0.08 \%$ & 0 & - & - \\
\hline Other sites & $1.02 \%$ & $3.15 \%$ & $2.89 \%$ & 13.915 & $\mathbf{0 . 0 0 1}$ \\
\hline *Each percentage refers to the percentage of all HAls during a single year. & \\
\hline
\end{tabular}

Analysis of the anatomical sites of CAls also indicated that lower respiratory tract infections were the most common during all three years (Table 3). Over time, lower respiratory tract infections, blood stream infections, surgical site infections, and eye/ear/nose/throat infections decreased significantly (all $P<$ 0.05). Correspondingly, there were increases in the percentage of urinary system infections, abdominal and digestive system infections, and skin and soft tissue infections (all $P<0.05$ ). 
Table 3

Anatomical sites of community-acquired infections from 2018 to $2020^{*}$

\begin{tabular}{|llllll|}
\hline Infection site & $\mathbf{2 0 1 8}$ & $\mathbf{2 0 1 9}$ & $\mathbf{2 0 2 0}$ & $\chi^{2}$ & $\boldsymbol{P}$ \\
\hline Lower respiratory tract & $49.83 \%$ & $52.65 \%$ & $43.06 \%$ & 44.024 & $<0.001$ \\
\hline Urinary system & $18.41 \%$ & $18.72 \%$ & $26.94 \%$ & 59.748 & $<0.001$ \\
\hline Abdomen and digestive system & $8.16 \%$ & $11.83 \%$ & $11.81 \%$ & 18.153 & $<0.001$ \\
\hline Skin and soft tissue & $6.73 \%$ & $5.37 \%$ & $7.27 \%$ & 7.446 & $\mathbf{0 . 0 2 4}$ \\
\hline Blood system & $6.12 \%$ & $4.73 \%$ & $4.44 \%$ & 6.526 & $\mathbf{0 . 0 3 8}$ \\
\hline Surgical site & $1.98 \%$ & $1.48 \%$ & $0.74 \%$ & 11.586 & $\mathbf{0 . 0 0 3}$ \\
\hline Eye/ear/nose/throat/mouth & $1.93 \%$ & $0.76 \%$ & $1.02 \%$ & 13.013 & 0.001 \\
\hline Upper respiratory tract & $1.21 \%$ & $0.92 \%$ & $0.83 \%$ & 1.585 & 0.453 \\
\hline Central nervous system & $0.94 \%$ & $1.24 \%$ & $0.60 \%$ & 5.094 & 0.078 \\
\hline Bone and joint & $0.55 \%$ & $0.16 \%$ & $0.37 \%$ & 4.821 & 0.090 \\
\hline Genital tract & $0.55 \%$ & $0.44 \%$ & $0.19 \%$ & 3.745 & 0.154 \\
\hline Otitis externa, otitis media & $0.50 \%$ & 0 & 0 & - & - \\
\hline Cardiovascular system & $0.33 \%$ & $0.36 \%$ & $0.42 \%$ & 0.208 & 0.901 \\
\hline Other sites & $2.76 \%$ & $1.32 \%$ & $2.31 \%$ & 11.768 & $\mathbf{0 . 0 0 3}$ \\
\hline *Each percentage refers to the percentage of all CAls during a single year. & \\
\hline
\end{tabular}

\section{Changes in infectious pathogens}

Analysis of the pathogens responsible for HAls indicated that Gram-negative species were the most common during all three years, and that Klebsiella pneumoniae, A. baumannii, and E. coli were the top three species (Table 4). Compared with 2018, there were greater percentages of fungal infections during 2019 and 2020. 
Table 4

Species responsible for hospital-acquired infections from 2018 to 2020

\begin{tabular}{|c|c|c|c|c|c|}
\hline 2018 & & 2019 & & 2020 & \\
\hline Species & n (\%) & Species & $\mathrm{n}(\%)$ & Species & n (\%) \\
\hline $\begin{array}{l}\text { Klebsiella } \\
\text { pneumoniae }\end{array}$ & $\begin{array}{l}155 \\
(15.72)\end{array}$ & $\begin{array}{l}\text { Klebsiella } \\
\text { pneumoniae }\end{array}$ & $\begin{array}{l}188 \\
(17.84)\end{array}$ & $\begin{array}{l}\text { Klebsiella } \\
\text { pneumoniae }\end{array}$ & $\begin{array}{l}222 \\
(17.73)\end{array}$ \\
\hline $\begin{array}{l}\text { Acinetobacter } \\
\text { baumannii }\end{array}$ & $\begin{array}{l}154 \\
(15.62)\end{array}$ & $\begin{array}{l}\text { Acinetobacter } \\
\text { baumannii }\end{array}$ & $\begin{array}{l}133 \\
(12.62)\end{array}$ & Escherichia coli & $\begin{array}{l}131 \\
(10.46)\end{array}$ \\
\hline Escherichia coli & $\begin{array}{l}139 \\
(14.10)\end{array}$ & Escherichia coli & $\begin{array}{l}125 \\
(11.86)\end{array}$ & $\begin{array}{l}\text { Acinetobacter } \\
\text { baumannii }\end{array}$ & $\begin{array}{l}130 \\
(10.38)\end{array}$ \\
\hline $\begin{array}{l}\text { Pseudomonas } \\
\text { aeruginosa }\end{array}$ & $\begin{array}{l}87 \\
(8.82)\end{array}$ & Candida albicans & $\begin{array}{l}100 \\
(9.49)\end{array}$ & Candida albicans & $\begin{array}{l}104 \\
(8.31)\end{array}$ \\
\hline $\begin{array}{l}\text { Stenotrophomonas } \\
\text { maltophilia }\end{array}$ & $\begin{array}{l}63 \\
(6.39)\end{array}$ & $\begin{array}{l}\text { Pseudomonas } \\
\text { aeruginosa }\end{array}$ & $\begin{array}{l}83 \\
(7.87)\end{array}$ & $\begin{array}{l}\text { Pseudomonas } \\
\text { aeruginosa }\end{array}$ & $\begin{array}{l}101 \\
(8.07)\end{array}$ \\
\hline $\begin{array}{l}\text { Enterobacter } \\
\text { cloacae }\end{array}$ & $\begin{array}{l}49 \\
(4.97)\end{array}$ & $\begin{array}{l}\text { Enterococcus } \\
\text { faecium }\end{array}$ & $\begin{array}{l}54 \\
(5.12)\end{array}$ & $\begin{array}{l}\text { Staphylococcus } \\
\text { aureus }\end{array}$ & $\begin{array}{l}65 \\
(5.19)\end{array}$ \\
\hline $\begin{array}{l}\text { Staphylococcus } \\
\text { aureus }\end{array}$ & $\begin{array}{l}45 \\
(4.56)\end{array}$ & $\begin{array}{l}\text { Staphylococcus } \\
\text { aureus }\end{array}$ & $50(4.74)$ & $\begin{array}{l}\text { Stenotrophomonas } \\
\text { maltophilia }\end{array}$ & $\begin{array}{l}56 \\
(4.47)\end{array}$ \\
\hline Candida albicans & $\begin{array}{l}38 \\
(3.85)\end{array}$ & $\begin{array}{l}\text { Stenotrophomonas } \\
\text { maltophilia }\end{array}$ & $\begin{array}{l}45 \\
(4.27)\end{array}$ & Candida smooth & $\begin{array}{l}54 \\
(4.31)\end{array}$ \\
\hline $\begin{array}{l}\text { Enterococcus } \\
\text { faecalis }\end{array}$ & $\begin{array}{l}35 \\
(3.55)\end{array}$ & Candida smooth & $\begin{array}{l}42 \\
(3.98)\end{array}$ & $\begin{array}{l}\text { Enterococcus } \\
\text { faecium }\end{array}$ & $\begin{array}{l}48 \\
(3.83)\end{array}$ \\
\hline Candida smooth & $\begin{array}{l}27 \\
(2.74)\end{array}$ & $\begin{array}{l}\text { Enterobacter } \\
\text { cloacae }\end{array}$ & $\begin{array}{l}41 \\
(3.89)\end{array}$ & $\begin{array}{l}\text { Enterobacter } \\
\text { cloacae }\end{array}$ & $\begin{array}{l}45 \\
(3.59)\end{array}$ \\
\hline others & $\begin{array}{l}194 \\
(19.68)\end{array}$ & others & $\begin{array}{l}193 \\
(18.31)\end{array}$ & others & $\begin{array}{l}296 \\
(23.64)\end{array}$ \\
\hline Total & $\begin{array}{l}986 \\
(100)\end{array}$ & Total & $\begin{array}{l}1054 \\
(100)\end{array}$ & Total & $\begin{array}{l}1252 \\
(100)\end{array}$ \\
\hline
\end{tabular}

Gram-negative bacteria were also responsible for most CAls during all three years, and E. coli, $K$. pneumoniae, A. baumannii, and P. aeruginosa were the top four species (Table 5). Compared with 2018, there were also higher percentages of fungal infections during 2019 and 2020, and a decline in the percentage of S. aureus infections from 8.96\% (2018), to 5.98\% (2019), and then to $4.32 \%$ (2020). 
Table 5

Species responsible for community-acquired infections from 2018 to 2020

\begin{tabular}{|c|c|c|c|c|c|}
\hline 2018 & & 2019 & & 2020 & \\
\hline Species & n (\%) & Species & $\mathrm{n}(\%)$ & Species & $\mathrm{n}(\%)$ \\
\hline Escherichia coli & $\begin{array}{l}247 \\
(17.85)\end{array}$ & Escherichia coli & $\begin{array}{l}366 \\
(19.38)\end{array}$ & Escherichia coli & $\begin{array}{l}356 \\
(16.71)\end{array}$ \\
\hline $\begin{array}{l}\text { Klebsiella } \\
\text { pneumoniae }\end{array}$ & $\begin{array}{l}190 \\
(13.73)\end{array}$ & $\begin{array}{l}\text { Klebsiella } \\
\text { pneumoniae }\end{array}$ & $\begin{array}{l}245 \\
(12.97)\end{array}$ & $\begin{array}{l}\text { Klebsiella } \\
\text { pneumoniae }\end{array}$ & $\begin{array}{l}323 \\
(15.16)\end{array}$ \\
\hline $\begin{array}{l}\text { Acinetobacter } \\
\text { baumannii }\end{array}$ & $\begin{array}{l}183 \\
(13.22)\end{array}$ & $\begin{array}{l}\text { Acinetobacter } \\
\text { baumannii }\end{array}$ & $\begin{array}{l}178 \\
(9.42)\end{array}$ & $\begin{array}{l}\text { Acinetobacter } \\
\text { baumannii }\end{array}$ & $\begin{array}{l}215 \\
(10.09)\end{array}$ \\
\hline $\begin{array}{l}\text { Pseudomonas } \\
\text { aeruginosa }\end{array}$ & $\begin{array}{l}135 \\
(9.75)\end{array}$ & $\begin{array}{l}\text { Pseudomonas } \\
\text { aeruginosa }\end{array}$ & $\begin{array}{l}154 \\
(8.15)\end{array}$ & $\begin{array}{l}\text { Pseudomonas } \\
\text { aeruginosa }\end{array}$ & $\begin{array}{l}162 \\
(7.61)\end{array}$ \\
\hline $\begin{array}{l}\text { Staphylococcus } \\
\text { aureus }\end{array}$ & $\begin{array}{l}124 \\
(8.96)\end{array}$ & candida albicans & $\begin{array}{l}149 \\
(7.89)\end{array}$ & Candida albicans & $\begin{array}{l}161 \\
(7.56)\end{array}$ \\
\hline Candida albicans & $\begin{array}{l}65 \\
(4.70)\end{array}$ & $\begin{array}{l}\text { Staphylococcus } \\
\text { aureus }\end{array}$ & $\begin{array}{l}113 \\
(5.98)\end{array}$ & $\begin{array}{l}\text { Staphylococcus } \\
\text { aureus }\end{array}$ & $\begin{array}{l}92 \\
(4.32)\end{array}$ \\
\hline $\begin{array}{l}\text { Stenotrophomonas } \\
\text { maltophilia }\end{array}$ & $\begin{array}{l}50 \\
(3.61)\end{array}$ & $\begin{array}{l}\text { Enterococcus } \\
\text { faecium }\end{array}$ & $\begin{array}{l}71 \\
(3.76)\end{array}$ & $\begin{array}{l}\text { Enterococcus } \\
\text { faecium }\end{array}$ & $\begin{array}{l}85 \\
(3.99)\end{array}$ \\
\hline $\begin{array}{l}\text { Enterococcus } \\
\text { faecium }\end{array}$ & $\begin{array}{l}48 \\
(3.47)\end{array}$ & $\begin{array}{l}\text { Enterococcus } \\
\text { faecalis }\end{array}$ & $\begin{array}{l}63 \\
(3.34)\end{array}$ & Enterobacter cloacae & $\begin{array}{l}72 \\
(3.38)\end{array}$ \\
\hline $\begin{array}{l}\text { Enterobacter } \\
\text { cloacae }\end{array}$ & $\begin{array}{l}48 \\
(3.47)\end{array}$ & Candida smooth & $\begin{array}{l}53 \\
(2.81)\end{array}$ & $\begin{array}{l}\text { Stenotrophomonas } \\
\text { maltophilia }\end{array}$ & $\begin{array}{l}64 \\
(3.00)\end{array}$ \\
\hline $\begin{array}{l}\text { Enterococcus } \\
\text { faecalis }\end{array}$ & $\begin{array}{l}28 \\
(2.02)\end{array}$ & $\begin{array}{l}\text { Enterobacter } \\
\text { cloacae }\end{array}$ & $\begin{array}{l}51 \\
(2.70)\end{array}$ & $\begin{array}{l}\text { Enterococcus } \\
\text { faecalis }\end{array}$ & $\begin{array}{l}60 \\
(2.82)\end{array}$ \\
\hline others & $\begin{array}{l}266 \\
(19.22)\end{array}$ & $\begin{array}{l}\text { Stenotrophomonas } \\
\text { maltophilia }\end{array}$ & $\begin{array}{l}46 \\
(2.44)\end{array}$ & Candida smooth & $\begin{array}{l}59 \\
(2.77)\end{array}$ \\
\hline Total & $\begin{array}{l}1384 \\
(100)\end{array}$ & Total & $\begin{array}{l}1889 \\
(100)\end{array}$ & Total & $\begin{array}{l}2130 \\
(100)\end{array}$ \\
\hline
\end{tabular}

\section{Changes in HAls by MDROs}

Analysis of HAls by MDROs indicated MRSA infections were more common in 2020 than in 2018 and 2019 (both $P<0.05$ ), but there were no significant changes in infections by VRE, CRE, CR-AB, or CR-PA (Table 6). 
Table 6

Hospital-acquired infections by the five main MDROs from 2018 to $2020^{*}$

\begin{tabular}{|llllllllllll|}
\hline \multirow{2}{*}{ Year } & \multicolumn{3}{l}{ MRSA } & \multicolumn{2}{l}{ VRE } & \multicolumn{2}{l}{ CRE } & \multicolumn{2}{l}{ CR-AB } & \multicolumn{2}{l|}{ CR-PA } \\
\cline { 2 - 13 } & $\mathbf{N}$ & $\%$ & $\mathbf{n}$ & $\%$ & $\mathbf{n}$ & $\%$ & $\mathbf{n}$ & $\%$ & $\mathbf{n}$ & $\%$ \\
\hline 2018 & 25 & $0.04 \%$ & 0 & 0 & 5 & $0.01 \%$ & 99 & $0.16 \%$ & 29 & $0.05 \%$ \\
2019 & 25 & $0.04 \%$ & 1 & $0.001 \%$ & 5 & $0.01 \%$ & 87 & $0.12 \%$ & 28 & $0.04 \%$ \\
2020 & 41 & $0.07 \%$ & 1 & $0.001 \%$ & 5 & $0.01 \%$ & 74 & $0.13 \%$ & 39 & $0.07 \%$ \\
$\chi^{2}$ & 9.038 & - & & - & & 3.779 & 4.813 \\
\hline
\end{tabular}

\section{Changes in use of different antibiotics}

We also determined the rate of antibiotic use as the percentage of all inpatients who received an antibiotic during each year (Table 7). The results indicated a significant decline over time (2018 vs. 2019: $P=0.001 ; 2018$ vs. 2020: $P<0.001 ; 2019$ vs. 2020: $P<0.001)$. There was also a significant increase in the culture rate of microbial specimens before administration of antibiotics (2018 vs. 2019: $P<0.001 ; 2018$ vs. 2020: $P<0.001 ; 2019$ vs. 2020: $P<0.001)$ and a significant decrease in the total days of antibiotic use based on Z values (2018 vs. 2019: -16.562; 2018 vs. 2020: $-22.682 ; 2019$ vs. 2020: $-6.670, P<0.001$ ). The AUD was relatively stable from 2018 to 2020 .

Table 7

Antibiotic administration practices from 2018 to 2020*

\begin{tabular}{|lllll|}
\hline Year & Overall (\%) & Culture before use (\%) & Average days (IQR) & AUD \\
\hline 2018 & 27.08 & 51.71 & $6(3,9)$ & 51.21 \\
\hline 2019 & 26.26 & 59.28 & $5(2,8)$ & 53.15 \\
\hline 2020 & 22.83 & 62.70 & $4(2,7)$ & - \\
\hline$\chi^{2}$ & 326.538 & 406.463 & 600.335 & - \\
\hline$P$ & $<0.001$ & $<0.001$ & $<0.001$ & \\
\hline $\begin{array}{l}* \\
\text { * 'Overall' is the percentage of all inpatients who received an antibiotic during a single year; 'Culture } \\
\text { the average duration of use among antibiotic users. }\end{array}$ \\
\hline \multicolumn{4}{|l}{ AUD: average use density; IQR: interquartile range. } \\
\hline
\end{tabular}


We further analyzed the AUD of the top five specific drugs, which were $\beta$-lactams, mainly cephalosporins (Table 8). Compared with 2018 and 2019, the AUD of special grade antibiotics against Gram-positive bacteria and fluoroquinolones was lower in 2020. Compared with 2018, the AUD of antifungal drugs was greater during 2019 and 2020.

Table 8

Antibiotic use density of the top ten antimicrobial agents from 2018 to 2020

\begin{tabular}{|c|c|c|c|c|c|}
\hline 2018 & & 2019 & & 2020 & \\
\hline Drug & AUD & Drug & AUD & Drug & AUD \\
\hline $\begin{array}{l}\text { Cefodizime sodium } \\
\text { injection }\end{array}$ & 10.22 & $\begin{array}{l}\text { Cefodizime sodium } \\
\text { injection }\end{array}$ & 10.93 & $\begin{array}{l}\text { Cefodizime sodium } \\
\text { injection }\end{array}$ & 9.8 \\
\hline Ceftizoxime sodium & 5.40 & Ceftizoxime sodium & 6.01 & $\begin{array}{l}\text { Cefoperazone and } \\
\text { sulbactam }\end{array}$ & 6.22 \\
\hline $\begin{array}{l}\text { Cefoperazone and } \\
\text { sulbactam }\end{array}$ & 4.04 & $\begin{array}{l}\text { Cefoperazone and } \\
\text { sulbactam }\end{array}$ & 4.84 & Ceftizoxime sodium & 6.10 \\
\hline $\begin{array}{l}\text { Cefazolin codium } \\
\text { pentahydrate injection }\end{array}$ & 3.76 & $\begin{array}{l}\text { Cefuroxime sodium } \\
\text { injection }\end{array}$ & 4.46 & $\begin{array}{l}\text { Cefuroxime sodium } \\
\text { injection }\end{array}$ & 4.75 \\
\hline $\begin{array}{l}\text { Cefuroxime sodium } \\
\text { injection }\end{array}$ & 3.17 & $\begin{array}{l}\text { Cefazolin sodium } \\
\text { pentahydrate injection }\end{array}$ & 3.37 & $\begin{array}{l}\text { Cefazolin sodium } \\
\text { pentahydrate } \\
\text { injection }\end{array}$ & 2.48 \\
\hline $\begin{array}{l}\text { Moxifloxacin } \\
\text { hydrochloride and } \\
\text { sodium chloride injection }\end{array}$ & 2.14 & $\begin{array}{l}\text { Moxifloxacin } \\
\text { hydrochloride and } \\
\text { sodium chloride } \\
\text { injection }\end{array}$ & 2.30 & $\begin{array}{l}\text { Meropenem } \\
\text { injection }\end{array}$ & 1.16 \\
\hline $\begin{array}{l}\text { Ornidazole and sodium } \\
\text { chloride injection }\end{array}$ & 1.73 & $\begin{array}{l}\text { Levofloxacin sodium } \\
\text { chloride injection }\end{array}$ & 2.01 & Biapenem & 1.04 \\
\hline $\begin{array}{l}\text { Levofloxacin sodium } \\
\text { chloride injection }\end{array}$ & 1.63 & $\begin{array}{l}\text { Linezolid glucose } \\
\text { injection }\end{array}$ & 1.66 & $\begin{array}{l}\text { Fluconazole and } \\
\text { sodium chloride } \\
\text { Injection }\end{array}$ & 1.03 \\
\hline Biapenem & 1.50 & Meropenem injection & 1.26 & $\begin{array}{l}\text { Moxifloxacin } \\
\text { hydrochloride and } \\
\text { sodium chloride } \\
\text { injection }\end{array}$ & 0.99 \\
\hline $\begin{array}{l}\text { Linezolid glucose } \\
\text { injection }\end{array}$ & 1.49 & Voriconazole & 1.23 & $\begin{array}{l}\text { Ornidazole and } \\
\text { sodium chloride } \\
\text { injection }\end{array}$ & 0.99 \\
\hline
\end{tabular}

\section{Discussion}

Infection prevention and control has played key roles in the fight against the COVID-19 pandemic, as indicated by the absence of any infections among the nearly 42,000 medical support staff in Hubei Province. The simultaneous increase in the public's awareness of infection prevention and control 
measures was also successful in preventing COVID-19 in the community [14]. Although China's domestic policies were successful, the pandemic is still a serious problem in many other countries, and China still has a risk that new cases will be imported. The "Normalized Epidemic Prevention and Control Requirements" measures of May 2020 have been key to the control of the COVID-19 pandemic [7, 8]. Based on risk classification, targeted measures should be taken, such as wearing masks in densely closed places. To prevent routine infection and improve infection control, hospitals have taken additional epidemic prevention and control measures, especially wearing masks and strengthening the regulations regarding individuals entering and exiting hospitals.

It is now more than 1 year after implementation of the "Normalized Epidemic Prevention and Control Requirements", and this study showed that the HAl rate in 2020 (1.82\%) was similar to the average HAI rate reported during the most recent five years in China (1.94\%) [15], but significantly higher than the HAl rates at our facility during 2018 and 2019. Although the CAl rates in 2020 and 2019 were significantly higher than in 2018, there was no difference between 2019 and 2020. Our results indicated the lower respiratory tract was the most common site of HAls, were similar to the results previously reported for Beijing [16] and in a 5-year national cross-sectional survey [17]. This may be because the respiratory tract is more vulnerable to HAls, and because it is easy to access the respiratory tract for collection of specimens and diagnosis. We found no significant differences in the percentage of lower respiratory tract HAls from 2018 to 2020.

From the perspective of epidemic prevention and control, measures such as wearing masks and maintaining a proper social distance are important for prevention of respiratory infectious diseases. However, the rates of HAls of the respiratory system did not change during our 3-year study period. In addition, measures such as fixed escort, restricted visits, and public education [18-19] can reduce the risk of cross-infection, but our results provided no evidence that they prevented HAls. There is a general consensus that increased hand hygiene compliance can reduce the incidence of HAls [20,21] and that overall use of alcohol-based hand cleaner is a reliable indicator of hand hygiene compliance [22, 23]. We found that the use of hand cleaner and average number of open beds per day increased in tandem from 2018 to 2020 , but this apparently had no impact on the rate of HAls.

Analysis of the causes and occurrence of HAls can be difficult [24] because of the impact of endogenous and exogenous factors. Thus, even if exogenous factors are controlled through prevention and control measures, the effects of endogenous factors may remain. Moreover, as a regional comprehensive medical center, our institution mostly admits emergency and critical patients, and a patient's condition determines the risk of a HAl, but the prevention and control measures at the hospital do not.

Our analysis of the anatomical sites of CAls indicated the lower respiratory tract was the most common site (as with HAls), but the percentage of lower respiratory tract infections was lower in 2020 than during the previous two years. This may be related to the implementation of the "Normalized Epidemic Prevention and Control Requirements" at the community level. In particular, the COVID-19 pandemic forced people to avoid unnecessary socialization, to wear masks, and to perform frequent hand washing 
to reduce the probability of cross-infection. However, our analysis of upper respiratory tract CAls indicated no significant change over time. This may be because most upper respiratory tract infections are caused by viruses, and these patients often recover without the need for hospitalization. Therefore, our percentage of CAls of the upper respiratory tract among inpatients (less than $2 \%$ ) was probably much lower than the percentage in all community-dwelling individuals.

The composition of pathogens responsible for HAls was relatively stable from 2018 to 2020 . Gramnegative bacteria accounted for nearly $60 \%$ of the top ten species, and $K$. pneumoniae ranked first; in contrast, other studies in China reported that $P$. aeruginosa ranked first among HAls $[15,17,25]$. This difference may be due to geographic differences. The pathogens responsible for CAls were similar to those responsible for HAls (i.e., mainly Gram-negative bacteria), but E. coli ranked first for CAls. This is probably because the urinary system was a much more common infection site for CAls. It is particularly noteworthy that proportion of fungal CAls and HAls increased from 2018 to 2020 . This may be due to the increase of opportunistic infections caused by the increasing incidence of tumor diseases and use of immunosuppressants [26]. Our analysis of MDROs indicated no significant changes, except that MRSA infection was significantly greater during 2020 than 2018 and 2019. This may be because HAls by MDROs mostly occur in the ICU [27], and most of these patients have acute and critical diseases, poor clinical status, and the measures of the "Normalized Epidemic Prevention and Control Requirements" had no impact in preventing infections by MDROs in this specific population.

The rational use of antibiotics is closely related to patient prognosis and the occurrence by antibioticresistant bacteria. The problem of drug-resistant bacteria has become a serious worldwide public health problem. The frequency of antibiotic use, the culture rate of microbial specimens, duration of antibiotic use, and AUD are all important indicators [28]. Our results showed that from 2018 to 2020, the rate and duration of use gradually decreased, and the rate of culturing before use had a gradual increase. Although the utilization rate decreased, administration of antibiotics to more than $20 \%$ of all inpatients was still very high because the sum of all HAls and CAls among inpatients was less than $5 \%$. At the same time, the significant declines in the utilization rate and days of utilization may be related to changes in Chinese medical insurance policies, in addition to the increased awareness by doctors of the problem of over-prescribing antibiotics. AUD is a key index used to assess the rationality of the long-term use of antibiotics. Previous studies confirmed that AUD is closely related to bacterial drug resistance [29, 30]. The present study showed that AUD increased slightly (but not significantly) from 2018 to 2020, consistent with the slight increases in HAls and CAls. Our analysis of the AUD of individual drugs indicated the AUDs of the five most common drugs (mostly cephalosporins) were relatively stable from 2018 to 2020, similar to the results of Yao et al. [31]. This may be because of the greater convenience and safety of these drugs, and the fewer restrictions regarding their use [32]. In addition, changes in the AUD of antibiotics may also be related to changes in infection sites, such as increasing proportion of infections by Gram-negative bacteria and fungi.

Of course, there are some limitations in this study. First, the study period was only 3 years, and may not be fully representative. Second, this study was conducted at a single center study, and the research area 
and scope need to be expanded to verify the generalizability of the conclusions.

\section{Conclusions}

We compared HAls and CAls in a study group (May to December 2020) with a control group (May to December of 2018 and 2019), and examined all inpatients from the same institution to reduce bias. We found that adoption of the "Normalized Epidemic Prevention and Control Requirements" on 1 May 2020 had no obvious impact on the overall rate of HAls nor on different parameters related to HAls.

\section{Abbreviations}

WHO:World Health Organization; COVID-19:Coronavirus disease 2019; SARS-CoV-2:Severe acute respiratory syndrome coronavirus 2; HAls:hospital-acquired infections; CAls:Community-acquired infections; CDC:Centers for Disease Control and Prevention; CLSI:Clinical Laboratory Standards Institute; MDROs:Multi-drug resistant organisms; MRSA:Methicillin-resistant Staphylococcus aureus; VRE:Vancomycin-resistant Enterococcus; CRE:Carbapenem-resistant Enterobacteriaceae; CRAB:Carbapenem-resistant Acinetobacter baumannii; CR-PA:Carbapenem-resistant Pseudomonas aeruginosa; AUD:Antibiotic use density; DDDs:defined daily doses; IQR:interquartile range.

\section{Declarations}

\section{Acknowledgments}

We thank the hospital staff for their efforts in recruiting patients.

\section{Authors'contributions}

Caiyun Chen and Ping Zhu have made substantial contributions to the conception and drafted the manuscript. Yongxiang Zhang substantively revised manuscript. Bo Liu have made substantial contribution to design of work and supervised protocol development. All authors have read and approved the final manuscript.

\section{Funding}

This work was done with support from the research on hospital management innovation program of Jiangsu Hospital Association in 2021 (JSYGY-3-2021-511). We thank Medjaden Inc. for scientific editing of this manuscript.

\section{Availability of data and materials}


After publication, the data generated in the current study will be available to others on reasonable request to the corresponding author.

\section{Ethics approval and consent to participate}

The methods were carried out in accordance with relevant guidelines and regulations. Ethical approval for this retrospective study was obtained from the local ethics committee of the First Affiliated Hospital with Nanjing Medical University (2021-SR-152). Informed informed consent was waived by the local ethics committee of the First Affiliated Hospital with Nanjing Medical University. It is not required to get administrative permission for accessing the raw data, and the data used in this study was anonymized before use.

\section{Consent for publication}

Not applicable.

\section{Conflicts of interest}

The authors declare that they have no conflicts of interest.

\section{References}

1. Carlo Contini, Mariachiara Di Nuzzo, Nicole Barp, et al. The novel zoonotic COVID-19 pandemic: An expected global health concern. J Infect Dev Ctries.2020;14(3):254-264.

2. World Health Organization. Coronavirus disease (COVID-19) outbreak (http://www.who.int).

3. Stephan Ludwig, Alexander Zarbock. Coronaviruses and SARS-CoV-2: A Brief Overview.Anesth Analg.2020;131(1):93-96.

4. Yuhang Wang, Matthew Grunewald, Stanley Perlman. Coronaviruses: An Updated Overview of Their Replication and Pathogenesis. Methods Mol Biol.2020; 2203:1-29.

5. Yan-Rong Guo, Qing-Dong Cao, Zhong-Si Hong, et al. The origin, transmission and clinical therapies on coronavirus disease 2019 (COVID-19) outbreak-an update on the status.Mil Med Res.2020;7(1):11. https://covid19.who.int/.Accessed 27 Aug 2021. Further notice on COVID-19 prevention and control work in key units, key populations and key places.Joint prevention and control mechanism of the State Council.2020.http://www.gov.cn/zhengce/content/2020 04/08/content_5500241.htm.Accessed 27 Aug 2021.Notice on implementing the requirements for normalized epidemic prevention and control requirements and further strengthening the infection prevention and control work in medical institutions.Joint prevention and control mechanism of the State Council.2020.http://www.gov.cn/xinwen/2020-05/01/content_5508135.htm.Accessed 27 Aug 2021.

6. Horan TC,Andrus M,Dudeck MA. CDC/NHSN surveillance definition of health care-associated infection and criteria for specific types of infections in the acute care setting.Am J Infect 
Control.2008;36(5):309-332.

7. Shang Hong, Wang Yusan, Shen Ziyu. National clinical laboratory operating procedures.4th ed. Beijing: People's Health Publishing House;2015.p.629-645.

8. Clinical Laboratory Standard Insttute (CLSI). Performance standards for antmicrobial susceptbility testng (2017) CLSI approved standards CLSI M100-S23.Wayne, PA: CLSI; 2017.

9. Huang Xun, Deng Zide, Ni Yuxing, et al. Chinese ecperts, consensus on prevention and control of multidrug resistance organism healthcare-associated infection.Chin J Infect Control, 2015;14(1): 19. 2008.03.002.

10. Rossi S, editor. Australian medicines handbook. Adelaide: Australian Medicines Handbook; Pty Ltd; 2017.

11. Yuxin Zhao, Sixiang Cheng, Xiaoyan Yu, et al.Chinese Public's Attention to the COVID-19 Epidemic on Social Media: Observational Descriptive Study.J Med Internet Res.2020;22(5): e18825.

12. LV Ling-fang, ZHANG Han-yang, ZHOU Chun-lian.Analysis of surveillance data of nosocomial infections in a level A tertiary hospital from 2014 to 2018.Chinese Journal of Disinfection.2020;37(5):355-358.

13. Hongwu Yao, Rui Huo, Yunxi Liu, et al. Incidence of healthcare-associated infections in a tertiary hospital in Beijing, China: results from a real-time surveillance system.Antimicrob Resist Infect Control.2019;8:145.

14. REN Nan, WEN Xi-mao, Wu An-hua. Nationwide cross-sectional survey on healthcare-associated infection in 2014. Chin J Infect Control.2016;15(2):83-87.

15. Kelly A Cawcutt, Jasmine R Marcelin, Julie K Silver.Using social media to disseminate research in infection prevention, hospital epidemiology, and antimicrobial stewardship.Infect Control Hosp Epidemiol.2019;40(11):1262-1268.

16. M Siracusa, S Scuri, I Grappasonni, et al.Healthcare acquired infections: malpractice and litigation issues. Ann lg.2019;31(5):496-506.

17. Sihem Ben Fredj, Asma Ben Cheikh, Sana Bhiri, et al. Multimodal intervention program to improve hand hygiene compliance: effectiveness and challenges.J Egypt Public Health Assoc.2020;95(1):11.

18. Jeneth Gutierrez, Aladeen Alloubani, Mohammad Alzaatreh, et al.Impact of an Interventional Program on Improving Compliance of Hand Hygiene and Reducing Hospital-Acquired Infection in the Critical Care Unit.J Glob Infect Dis.2021;13(2):80-84.

19. W Wetzker, K Bunte-Schönberger, J Walter, et al. Use of ventilator utilization ratio for stratifying alcohol-based hand-rub consumption data to improve surveillance on intensive care units.J Hosp Infect.2017;95(2):185-188.

20. Tobias Siegfried Kramer, Janine Walter, Christin Schröder, et al.Increase in consumption of alcoholbased hand rub in German acute care hospitals over a 12 year period.BMC Infect Dis. 2021;21(1):766. 
21. Kathryn R Dalton, Clare Rock, Karen C Carroll, et al.One Health in hospitals: how understanding the dynamics of people, animals, and the hospital built-environment can be used to better inform interventions for antimicrobial-resistant gram-positive infections.Antimicrob Resist Infect Control.2020;9(1):78.

22. SHENG Bo, YE Yin, LI Jiabin. Nosocomial infection: an analysis and investigation of inpatients cases.Anhui Medical and Pharmaceutical Journal. 2017;21(1):77-81.

23. Balaji Pathakumari, Guanzhao Liang, Weida Liu.Immune defence to invasive fungal infections: A comprehensive review.Biomed Pharmacother.2020;130:110550.

24. Jan J De Waele, Jerina Boelens, Isabel Leroux-Roels, et al.Multidrug-resistant bacteria in ICU: fact or myth.Curr Opin Anaesthesiol.2020;33(2):156-161.

25. Styliani Karanika, Suresh Paudel, Christos Grigoras, et al.Systematic Review and Meta-analysis of Clinical and Economic Outcomes from the Implementation of Hospital-Based Antimicrobial Stewardship Programs.Antimicrob Agents Chemother.2016;60(8):4840-52.

26. Luminita Baditoiu, Carmen Axente, Diana Lungeanu, et al.Intensive care antibiotic consumption and resistance patterns: a cross-correlation analysis.Ann Clin Microbiol Antimicrob.2017;16(1):71.

27. Milan Kolar, Miroslava Htoutou Sedlakova, Karel Urbanek, et al.Implementation of Antibiotic Stewardship in a University Hospital Setting. Antibiotics (Basel). 2021;10(1):93.

28. Yao L, Zhang R, Mao S, et al. Use of antibiotics in Shanghai second and third level hospitals during the period of 2007-2008. Eval Analyst Drug Hosp China.2010;10:971-3.

29. Ertugrul Guclu, Aziz Ogutlu, Oguz Karabay, et al. Antibiotic consumption in Turkish hospitals; a multicentre point prevalence study. J Chemother.2017;29(1):19-24.

\section{Figures}




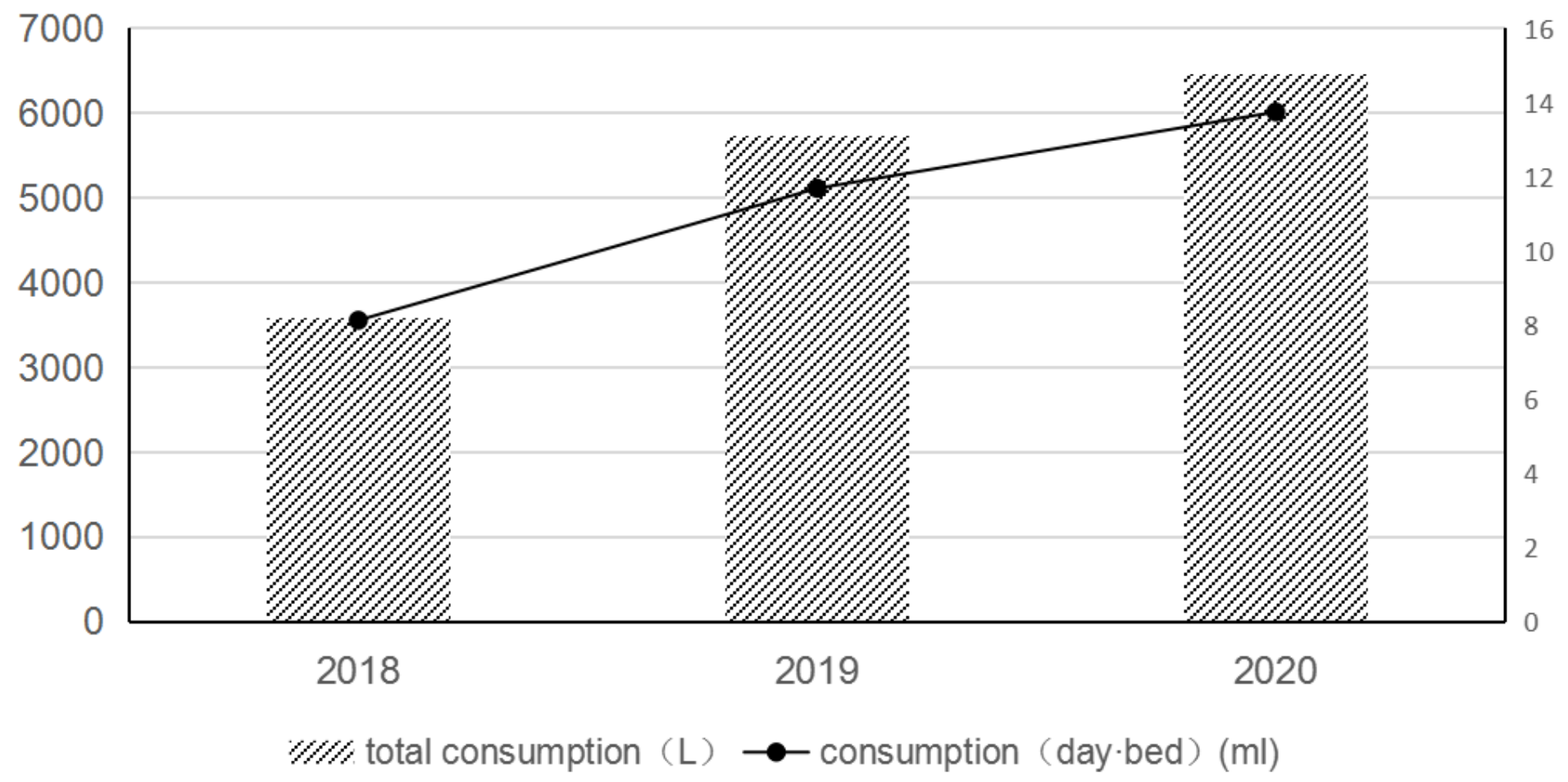

Figure 1

Use of alcohol-based hand cleaner (left axis) and average number of open beds per day (right axis) from 2018 to 2020 